International Journal of Wireless \& Mobile Networks (IJWMN) Vol. 8, No. 4, August 2016

\title{
Multi-Stages Co-OPERATIVE/NON- COOPERATIVE SCHEMES OF SPECTRUM SENSING FOR COGNITIVE RADIO SYSTEMS
}

\author{
Anwar Mousa $^{1}$ and Tara Javidi ${ }^{2}$ \\ University of California, San Diego (UCSD)- Jacobs School of Engineering \\ 9500 Gilman Dr., La Jolla, CA 92093-0407
}

\begin{abstract}
Searching for spectrum holes in practical wireless channels where primary users experience multipath fading and shadowing, with noise uncertainty, limits the detection performance significantly. Moreover, the detection challenge will be tougher when different band types have to be sensed, with different signal and spectral characteristics, and probably overlapping spectra. Besides, primary user waveforms can be known (completely or partially) or unknown to allow or forbid cognitive radios to use specific kinds of detection schemes! Hidden primary user's problem, and doubly selective channel oblige the use of cooperative sensing to exploit the spatial diversity in the observations of spatially located cognitive radio users. Incorporated all the aforementioned practical challenges as a whole, this paper developed a new multistage detection scheme that intelligently decides the detection algorithm based on power, noise, bandwidth and knowledge of the signal of interest. The proposed scheme switches between individual and cooperative sensing and among featured based sensing techniques (cyclo-stationary detection and matched filter) and sub-band energy detection according to the characteristics of signal and band of interest.Compared to the existing schemes, performance evaluations show reliable results in terms of probabilities of detection and mean sensing times under the aforementioned conditions.
\end{abstract}

\section{Keywords}

spectrum sensing,local and cooperative,cognitive radio, sub-band energy detection, probability of detection, mean detection time

\section{INTRODUCTION AND RELATED WORK}

Spectrum sensing can be individual into (non-cooperative) or cooperative [1]. In individual sensing, each cognitive radio (CR) performs spectrum sensing locally on the received signal and makes a decision about the presence or absence of a primary user (PU). However,in cooperative sensing, CRs perform individual sensing and direct their decisions or sensed information to a fusion center, and a final cooperative decision is taken at the fusion center. Hence, to increase the effectiveness of cooperative performance, it is necessary first to improve individual sensing. 
Individual sensing for single CR user, can be generallyclassified as matched filter detection, energy detection, cyclostationary feature detection, wavelet detection, multi antenna based sensing, eigenvalue based sensing and sub-Nyquist wideband based sensing.A matched filter (MF) is optimal to signal detection in the presence of AWGN as it maximizes the received signal to noise ratio (SNR) and takes minimum sensing time. That isthanks to a complete knowledge of primary user signal that should be available to the MF detector. If CR is operating in few PU bands, then MF is the best choice, but if the number of operating PU bands will increase, then practically, it is difficult to use MF because dedicated circuitry is essential for each PU licensee to achieve synchronization.Conventional Energy Detection (ED) is generally adopted for spectrum sensing because it does not need a priori information of the primary signal and enjoys low computational and implementation complexities.However, one of the major shortcomings of the ED is its poor performance when SNR falls below a certain threshold known as the SNR wall,that depends on noise uncertainty. However, Sub-band Based Energy Detection (SBED) [2] uses spectrum analysis techniques that make it possible to detect rapidly the spectral holes in a wide frequency band for CR operation. The most basic and computationally effective technique for spectrum analysis is block-wise FFT or AFB (analysis filter bank) processing of the observed signal and measuring the power of each sub-band. From the sensing point of view, when the channel is wide and frequency selective, AFB divides it into small and flat sub-band and optimized weighting process can be added to combat the effect of frequency selectivity, making it possible for reliable sensing even in low SNIR.

Cyclostationary feature detection (CS) has capability to isolate noise from useful signal, so it can work well under low SNR but requires some prior information of the primary user signal[3]. On the other hand, it suffers from high computational complexity and long sensing time. Wavelet detection is efficient for wideband signal but suffers also from high computational complexity. Eigenvalue based sensing does not need noise variance information and is considered as a possible solution to the challenging noise uncertainty conditions. Likewise, multi antenna based sensing, utilizing spatial correlations of PU signals, is considered an alternate method to afford robustness against the noise uncertainty effects, however, it suffers from increased hardware and computational complexity. Sub-Nyquist wideband based sensing is based on compressive sensing and multichannel sub-nyquist sampling techniques [4]. It refers to the procedure of employing sampling rates lower than the Nyquist rate and detecting spectrumholes using these partial measurements.

SNR-based two-stage adaptive spectrum sensing is proposed in [5]. In the first stage, the SNR is estimated for available channels. The CR then performs either ED or one-order CS detection based on the SNR estimated in the first stage of PU detection. Simulation results showed that reliable results can be attained with less mean detection time. In [6] the authors proposed a spectrum sensing scheme which obtains reliable results with less mean detection time. First, the scheme determines a better matched filter, or a combination of ED and CS based on the power and band of interest. An ED with a bi-threshold is used, and the CSdetector is applied only if the energy of the signal lies between two thresholds. Second, sensing is performed by the selection choice resulting from the first step. The distinction of the proposed scheme is that it deals with multiple types of primary systems, i.e., for PUs with known and unknown waveforms. However, all the existing two-stage detection schemes in the literature only considered single type of primary system. Similarly, in [7] an adaptive local spectrum sensing scheme is proposed. First, 
the channels available in the bandwidth of interest are sensed serially. The scheme determines a better MF, or a combination of ED and CSdetectors based on the available information of the signal present in the channel. The concept of SNR wall was also discussed for ED. One-order CS detection is performed in time domain in place of CS in frequency domain so that real-time operation and low-computational complexity can be accomplished. Likewise, a two-stage spectrum sensing scheme is also proposed in [8], in which the ED is used at the first stage to sort channels in ascending order based on the power of each. The one-order CS is used on the channel with the lowest power to detect weak signals in the second stage. The authors [9] in proposed two novel schemes of two stage spectrum sensing for $\mathrm{CR}$ under environment as noise power uncertainty. The two-stage spectrum sensing technique combines two conventional spectrum sensing methods to perform sensing by exploiting their individual advantages. However, in [10], authors proposed a two-stage fuzzy logic-based sensing where the output from each technique employed in the first stage is combined in the second stage using a fuzzy logic to make the final decision. Likewise, [11] proposed high-speed two-stage detector based on an ED. If the measured energy is greater than a specific threshold then PU present is decided in the first stage, else SNR is computed where if it is greater than another SNR threshold, then the result is still valid. Otherwise, then second stage is performed based on covariance absolute value. In [12] an ED is used in the first stage to estimates the SNR, based on which, it declares the absence or presence of a PU at the first stage, otherwise it runs the second stage to have aprecise decision.

Noise uncertainty, multipath fading and shadowing, which are characteristics of practical wireless channels, degrade the detection performance in individual spectrum sensing significantly. As an alternative solution to overcome the challenges of practical environments, cooperative sensing has been broadly studied in the literature as a method to improve the sensing performance. Besides, Hidden PU problem, which appears when the PU is not detectable by the sensing station, e.g., due to shadowing, can be solved [13]. The main idea of cooperative sensing is to enhance the sensing performance by using the spatial diversity in the observations of spatially located CR users.By cooperation, CR users can share their sensing information for making a combined decision more precise than the individual decisions. In [14],the authors considered a multichannel CR network, where cooperative CRs have heterogeneous sensing ability in terms of their sensing accuracy. They employed a group-based cooperative sensing scheme in which cooperating CRs are grouped such that different groups are responsible for sensing different channels. The performance enhancement due to spatial diversity is called cooperative gain. On the other hand, the cooperation overhead denotestheadditional sensing time, delay, energy, and operations dedicated to cooperative sensing, compared to the individual (non-cooperative) spectrum sensing case[15]. More specifically, the delay overheads in cooperative sensing are addressed as:

- Sensing delay, depends on the employedsensing method. The sensing time is proportional to the number of samples used by the signal detector where the longer the sensing time is, the better the performance will be. However, due to the hardware restriction that a single RF transceiver exists in each CR equipment, user cannot perform detection and transmissions in the same time. The more time is dedicated to sensing, the less time is available for transmissions and thus reducing the CR user throughput. This is known as the sensing efficiency problem [16] or the sensing-throughput tradeoff in spectrum sensing. In[17], the sensing-throughput tradeoff is formulated as an optimization problem to maximize the average CR throughput under the presence and the absence of PUs. 
International Journal of Wireless \& Mobile Networks (IJWMN) Vol. 8, No. 4, August 2016

- Reporting delay, in cooperative sensing, sharing local sensing data with other CR users and/or the FC (Fusion Center) yields reporting delay. This is part of cooperation overhead as it does not exist in non-cooperative spectrum sensing. In addition to transmission delay from the cooperating $\mathrm{CR}$ users to the FC, there are many causes that result in reporting delay. First, if cooperating $\mathrm{CR}$ users transmit on a control channel by a random access scheme, it is probable that the control messages sent from different CR users collide and then retransmission is requested. Besides, sending the sensing data by multiple hops, as the case in the relay-assisted cooperative sensing yields extra reporting delay. In[18], the authors addressed the issue of cooperation processing tradeoff in cooperative sensing. The tradeoff is formulated as an optimization problem to minimize the total sensing time subject to constraints of detection and false alarm probabilities.

- Synchronization delays, the synchronization of all the cooperating users is needed in many cooperative sensing schemes that depend on simultaneous reporting of the CR users. However, the synchronization may not be easily accomplished for a large amount of CR users. Hence, many asynchronous cooperative sensing methods are proposedin [19] to treat this problem.

\subsection{Problem Statement}

Searching for spectrum holes in practical wireless channels where PU's experience multipath fading and shadowing, with noise uncertainty, limits the detection performance significantly.The detection challenge will be tougher when different band types have to be sensed, with different signal and spectral characteristics, and possibly overlapping spectra. Each band can be narrow or wide, flat or frequency selective, experiencing AWGN or Rayleigh channel. Besides, PU waveforms can be known (completely or partially) or unknown to allow or forbid CRs to use specific kinds of detection schemes! Moreover, Hidden PU problem, and doubly selective channel oblige the use of cooperative sensing and exploit the spatial diversity in the observations of spatially located CR users.

However, according to the author's best knowledge, none of the existing approaches in literature have incorporated all the aforementioned practical challenges as a whole; all the existing multistage detection schemes in the literature only considered single type of primary system with single type of channel condition!

The proposed solutions to the above challenges will focus on:

- Developing adaptive Co-operative/non-cooperative multi-stage schemes of spectrum sensing. Cooperative sensing has been widely studied in the literature as a method to combat effects of multipath fading and hidden PU problem by exploiting space diversity of the PU's.

- Switching between individual and cooperative sensing and switching amongfeatured based sensing techniques (cyclo-stationary detection and matched filter)andSubband Energy Detection according to the characteristics of signal and band of interest. 
Hence, the distinction of the proposed multi-stage detection scheme is that it deals with: First,almost alltypes of PUs' signals and bands of interest. Second, it tries to detect signals experiencing practical wireless channels. Third, the proposed multi-stage scheme intelligently decides the detection algorithm based on the power, noise, bandwidth and knowledge of signal of interest, thus increasing accuracy and reducing mean detection time for the overall detection process. The remainder of this article is organized as follows. Section 2details system model and the proposed new adaptive multi-stage scheme. Section 3describes the considered setting and simulation parameters. Section 4 analyzes the achieved numerical results and Section 5 concludes the paper.

\section{System Model and Proposed New Adaptive Multi-Stage Scheme}

\subsection{System Model}

We consider $M$, CRs trying to detect $K$, PUs of different signal types, with different spectral characteristics, and possibly overlapping spectra, under various channel conditions. Each band can be narrow or wide, flat or frequency selective, experiencing AWGN or Rayleigh channel. The $M$, CRs and the $K$, PUs are spread in a coexisting coverage area. A blocking objects may exist to make hidden PUs to some CRs where some CR acts as relay to detect hidden ones. Some CRs acts as fusion center for cooperation sensing. To decrease hardware complexity of CRs, every $\mathrm{CR}$, rather than the fusion center, is equipped with only one specific type of detector. If the adaptive model chooses a specific type of detection, then the fusion center will order the CR which is equipped with that detector to perform sensing. Figure 1 shows the model for $M=4$ and $K=8$ where CR- 1 is a fusion center.

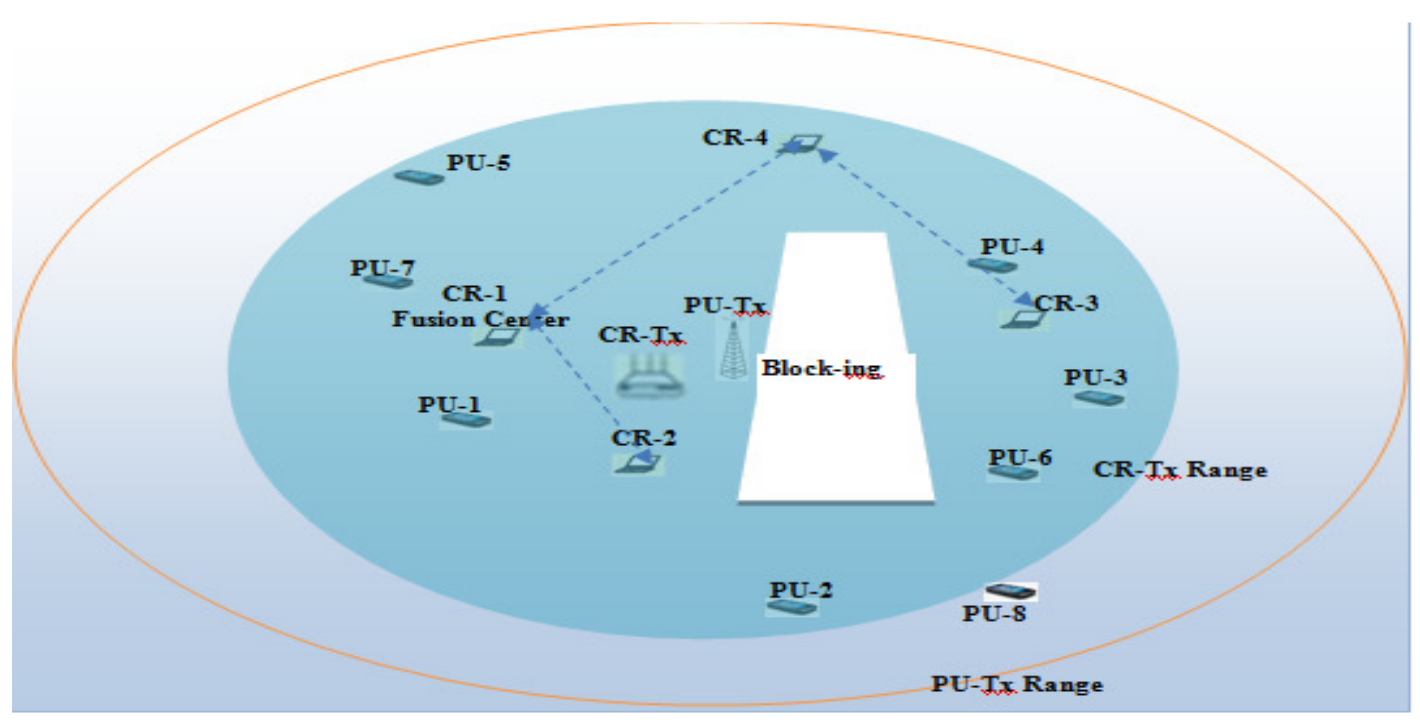

Figure 1: System Model 
International Journal of Wireless \& Mobile Networks (IJWMN) Vol. 8, No. 4, August 2016

\subsection{Proposed New Adaptive Multi-Stage Scheme}

The proposed scheme is shown in Figure 2. the CRs will perform comprehensive test of the observed signal of interest beginning bymeasuring the SNIR. In the first stage, the scheme will check whether the SNIR value of the PU signal of interest is greater than or equal to the SNIR wall, $\gamma_{\text {wall }},[20]$. If so, the scheme will perform non-cooperative sensing. Otherwise cooperative sensing is performed. If the SNIR of PU signals is below the SNR wall, the detection performance cannot be improved by increasing the sensitivity. Fortunately, thesensitivity requirement and the hardware limitation issuescan be considerably relieved by cooperative sensing.

In the second stage, the scheme checks whether the observed signal is wideband or narrow band. If narrow band signal and non-cooperative, the scheme will check again in a third stage whether complete or even partial knowledge of PU waveform is available to perform matched filter (MF) detection. The intuition behind the MF relies on the prior knowledge of a PU waveform, such as modulation type, order, the pulse shape, and the packet format. If this is true, then MF detection is performed as local (non-cooperative) decision. No need to pass thru the fourth stage since the threshold T is less than $\gamma_{\text {wall }}$ and the SNIR already exceeds $\gamma_{\text {wall. }}$. However, if the PU waveform is unknown, then the third stage will work in performing sub-band energy detection(SBED) under non-flat spectral characteristics as local (non-cooperative) decision. SBED, based on FFT/AFB, works for both wide and narrow band signals[2]. In the second stage, if the observed signal is wideband and non-cooperative, the scheme will also perform sub-band energy detection as this sensing method is suitable for wideband signals.

On the other hand, if the SNIR $<\gamma_{\text {wall }}$, then cooperative sensing is chosen, and the second stage will test the wideness of the signal band. If it is wide, then cooperative SBED is performed and the result is sent to the fusion center. On the other hand, if the signal band is narrow, then a third stage test for the signal is performed by checking whether complete or even partial knowledge of PU waveform is available to perform cooperative MF detection. If the signal is known enough, then a fourth stage test is carried out to ensure that the signal SNIR is greater than a threshold $T_{m f}$. The intuition behind the threshold $T_{m j}$ is to ensure that the SNIR is adequate for good performance of the MF. Note that the performance of the MF is relatively poor below certain SNIR[21]. If so, then cooperative MF detection is performed and the result is sent to the fusion center. If not, then cooperative one-order cyclo-statioary(OOCS) detection is performed. One-order cyclo-stationary detection is chosen although the performance of higher order cyclo-stationary detection is a bit better than that of one-order detection. The gain of higher orders is due to hardware complexity and power consumed by additional multiplying algorithm[22]. For commercial implementation of CRs, it is necessary to minimize hardware complexity and power consumption. Therefore, the OOCS detection will be used instead of the higher-order cyclo-stationary detection. On the other hand, if the PU waveform is unknown, then cooperative OOCS detection is also performed and the result is sent to the fusion center. 
International Journal of Wireless \& Mobile Networks (IJWMN) Vol. 8, No. 4, August 2016

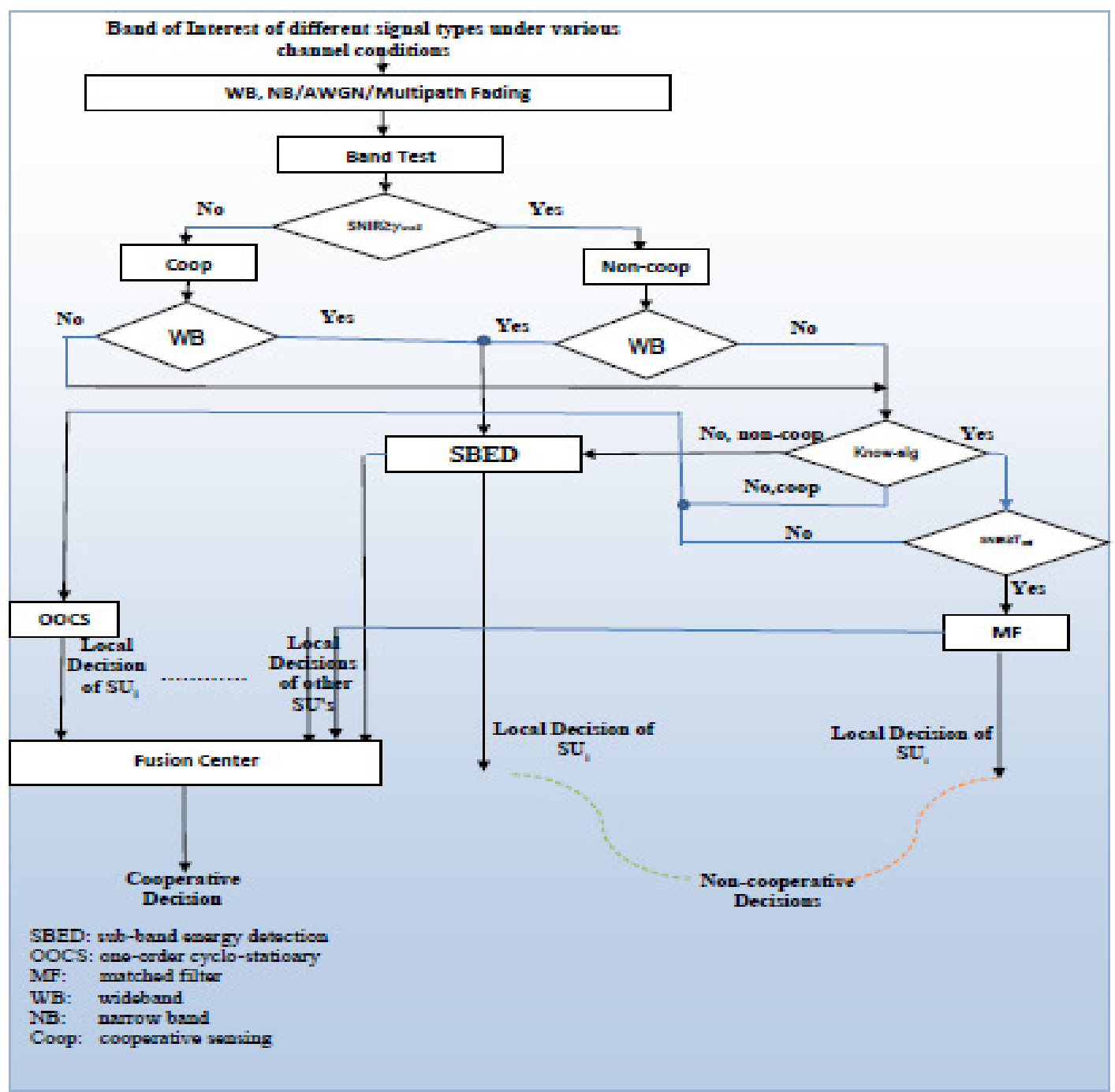

Figure 2: A Proposed Adaptive Sensing Model

\subsection{Performance Metrics}

To evaluate the scheme's performance, the results will be partiallycompared with those where only one type of detector exists. That is because no specific detector can accommodate different band types with different signal, channel and spectral characteristics as the proposed adaptive multi-stage scheme does. The performance metrics are the probability of detection, probability of false alarm, and mean overall detection time.

- Probability of detection $P_{d}$, shown as $(H 1 / H 1)$ i.e. probability of successful decision of the spectrum sensing process. Actually it confirms the presence of PU signal in a channel on the basis of decision of the spectrum sensing schemes. 
- Probability of false alarm $P_{f}$, shown as $(H 1 / H 0)$ i.e. probability of unsuccessful and false decision of the spectrum sensing process. In other words, it shows that PU signal is present in a channel while the channel is vacant.

- Mean overall detection time $\bar{T}$, defined as the mean time for detecting all available bands with different detectors, wither individually or cooperatively in the proposed multi-stage scheme.

Knowing that false alarms reduce spectral efficiency and miss detection causes interference with the PU, it is vital for optimal detection performance that maximum probability of detection is accomplished subject to minimum probability of false alarm. Relative to IEEE802.22 standard Error! Reference source not found., $P_{f}$ must be $<=0.1$ and $P_{d}>=0.9$.

The overall $P_{d}$ and $P_{f}$ of the proposed scheme, Figure 2, are derived as follow:

$P_{d}=P_{1} P_{d, n c o}+\left(1-P_{1}\right) P_{d, c o}$

$$
P_{f}=P_{1} P_{f, n c o}+\left(1-P_{1}\right) P_{f, c o}
$$

$P_{1} i s$ the probability that channel would be sensed by the non-cooperative detections(probability that $\left.\mathbf{S N R} \geq \gamma_{\text {wall }}\right)$, hence $\left(1-P_{1}\right)$ is the probability that channel would be sensed by the cooperative detection. $P_{d, n c o}$ and $P_{d, c o}$ are the probabilities for non-cooperative and cooperative detectionsrespectively. Similarly, $P_{f, n c o}$ and $P_{f, c o}$ are the probabilities for non-cooperative and cooperative false alarm respectively.

$$
\begin{gathered}
P_{d, c o}=P_{2} P_{d, E D}+\left(1-P_{2}\right) P_{d, c o-N B} \\
P_{f, c o}=P_{2} P_{f, E D}+\left(1-P_{2}\right) P_{f, c o-N B}
\end{gathered}
$$

$P_{2} i s$ the probability that the PU signal in interest is wideband, hence $\left(1-P_{2}\right)$ is the probability that the PU signal in interest is narrowband. $P_{d, E D}$ and $P_{f, E D}$ are the probabilities of detection and of false alarm, for energy detector, respectively. $P_{d, c o-N B}$ and $P_{f, c o-N B}$ are the probabilities of detection and of false alarm, for cooperative detections when the signal is narrowband, respectively.

$$
\begin{aligned}
& P_{d, n c o}=P_{2} P_{d, n c o-W B}+\left(1-P_{2}\right) P_{d, n c o-N B} \\
& P_{f, n c o}=P_{2} P_{f, n c o-W B}+\left(1-P_{2}\right) P_{f, n c o-N B}
\end{aligned}
$$

$P_{d, n c o-W B}$ and $P_{f, n c o-W B}$ are the probabilities of detection and of false alarm, for non-cooperative detections when the signal is wideband, respectively. 
Similarly, $P_{d, n c o-N B}$ and $P_{f, n c o-N B}$ are the probabilities of detection and of false alarm, for noncooperative detections when the signal is narrowband, respectively.

$$
\begin{aligned}
& P_{d, n c o-N B}=P_{3} P_{d, M F}+\left(1-P_{3}\right) P_{d, E D} \\
& P_{f, n c o-N B}=P_{3} P_{f, M F}+\left(1-P_{3}\right) P_{f, E D}
\end{aligned}
$$

$P_{d, M F}$ and $P_{f, M F}$ are the probabilities of detection and of false alarm, for matched filter, respectively. $P_{3}$ is the probability of complete knowledge of PU signal, yielding matched filter detection and $\left(1-P_{3}\right)$ is the probability of unknown PU signal.

$$
\begin{aligned}
& P_{d, n c o-W B}=P_{d, E D} \\
& P_{f, n c o-W B}=P_{f, E D} \\
& P_{d, c o-N B}=P_{3} P_{4} P_{d, M F}+\left(1-P_{3}\right) P_{d, C Y C}+P_{3}\left(1-P_{4}\right) P_{d, C Y C} \\
& P_{f, c o-N B}=P_{3} P_{4} P_{f, M F}+\left(1-P_{3}\right) P_{f, C Y C}+P_{3}\left(1-P_{4}\right) P_{f, C Y C}
\end{aligned}
$$

Here, $P_{d, c o-N B}$ and $P_{f, c o-N B}$ are the probabilities of detection and of false alarm, for cooperative detections when the signal is narrowband, respectively. $P_{4}$ is the probability that the SNIR is greater than $\mathrm{T}$, yielding cooperative matched filter detection of PU signal and $\left(1-P_{4}\right)$ return the PU signal for cooperative cyclo-statioarydetection .

The overall probability of detection and probability of false alarm of the proposed scheme become:

$$
P_{d}=P_{1}\left(P_{2} P_{\alpha, E D}+\left(1-P_{2}\right) P_{3} P_{\alpha, M F}+\left(1-P_{2}\right)\left(1-P_{3}\right) P_{\alpha, E D}\right)+\left(1-P_{1}\right)\left(P_{2} P_{\alpha, E D}+\left(1-P_{2}\right)\left[P_{3} P_{4} P_{\alpha, M F}+\left(1-P_{3}\right) P_{\alpha, C Y C}+P_{3}\left(1-P_{4}\right) P_{\alpha, C Y C}\right]\right)
$$

$$
P_{f}=P_{1}\left(P_{2} P_{f, E D}+\left(1-P_{2}\right) P_{3} P_{f, M F}+\left(1-P_{2}\right)\left(1-P_{3}\right) P_{f, E D}\right)+\left(1-P_{1}\right)\left(P_{2} P_{f, E D}+\left(1-P_{2}\right)\left[P_{3} P_{4} P_{f, M F}+\left(1-P_{3}\right) P_{f, C Y C}+P_{3}\left(1-P_{4}\right) P_{f, C Y C}\right]\right)
$$

The overall mean detection time $\bar{T}$ of the proposed sensing scheme is:

$\bar{T}=\overline{T_{n c o}}+\overline{T_{c o}}$

$\overline{T_{c o}}=\overline{T_{C Y C L O}}+\overline{T_{M F-C O}}+\overline{T_{E D-C O}}+\overline{T_{R}}+\overline{T_{S}}$

$\overline{T_{n c o}}=\overline{T_{M F-N C O}}+\overline{T_{E D-N C O}}$

Where $\overline{T_{n c o}}$ and $T_{c o}$ are the mean detection times for non-cooperative and cooperative detections respectively. $\overline{T_{M F-C O}}, \overline{T_{E D-C O}}$ and $\overline{T_{C Y C L O}}$ are the sensing times of cooperative matched filter detection, cooperative energy detection and one-order cyclostationary detection, respectively. 
Similarly, $\overline{T_{M F-N C O}}, \overline{T_{E D-N C O}}$ are the sensing times of non-cooperative matched filter detection and non-cooperative energy detection. In case of cooperative sensing, $\overline{T_{R}}$ is the reporting delay and $\overline{T_{S}}$ is the synchronization delay.

$\overline{T_{\text {cyclo }}}$ is derived as follows:

$$
\begin{aligned}
& \overline{T_{\text {cyclo }}}=N T_{\text {cyclo-1 }}\left[\left(1-P_{1}\right)\left(1-P_{2}\right)\left(1-P_{3}\right)+\left(1-P_{1}\right)\left(1-P_{2}\right) P_{3}\left(1-P_{4}\right)\right] \\
& \overline{T_{\text {cyclo }}}=N T_{\text {cyclo-1 }}\left[\left(1-P_{1}\right)\left(1-P_{2}\right)\left(1-P_{3} P_{4}\right)\right]
\end{aligned}
$$

where $T_{\text {cyclo-1 }}$ is the mean sensing time for each channel by the one-order cyclostationarydetector. $T_{c y c l o-1}=\frac{M_{C 1}}{2 W_{c 1}}$ in which $\mathrm{M}_{\mathrm{C} 1}$ is the number of samples for detection, and $W_{c 1}$ is the channel bandwidth. Similarly,

$\overline{T_{M F-C O}}$ and $\overline{T_{E D-C O}}$ are derived as follows:

$$
\begin{aligned}
& \overline{T_{M F-C O}}=N T_{M F-1}\left[\left(1-P_{1}\right)\left(1-P_{2}\right) P_{3} P_{4}\right] \\
& \overline{T_{E D-C O}}=N T_{E D-1}\left[\left(1-P_{1}\right) P_{2}\right]
\end{aligned}
$$

where $T_{M F-1}$ is the mean sensing time for each channel by the matched filter detector. $T_{M F-1}=\frac{M_{m f}}{2 W_{m f}}$ in which $M_{m f}$ is the number of samples for detection, and $W_{m f}$ is the channel bandwidth. $T_{E D-1}$ is the mean sensing time for each channel by the energy detector. $T_{E D-1}=\frac{M_{e d}}{2 W_{e d}}$ in which $M_{e d}$ is the number of samples for detection, and $W_{e d}$ is the channel bandwidth.

Likewise,

$$
\begin{aligned}
& \overline{T_{M F-N C O}}=N T_{M F-1}\left[P_{1}\left(1-P_{2}\right) P_{3}\right] \\
& \overline{T_{E D-N C O}}=N T_{E D-1}\left[P_{1} P_{2}+P_{1}\left(1-P_{2}\right)\left(1-P_{3}\right)\right]
\end{aligned}
$$

The overall mean detection time of the proposed scheme:

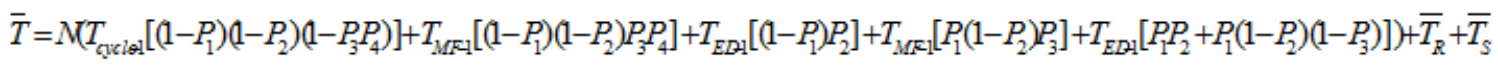


The following two cases can be made on the basis of $P_{1}$, the probability that $\mathrm{SNR} \geq \gamma_{\text {wall }}$.

Case 1: When $0 \leq P_{1}<0.5$ for the majority of the channels,SNIR $<\gamma_{\text {wall }}$. Therefore, the CRwill perform cooperative detection for sensing the majority of the channels because cooperative sensing is an efficient approach to combat multipath fading and shadowing and mitigate the receiver uncertainty problem detection encounteredat low SNR. When $P_{1} \approx 0$, then the probability of detection, the probability of false alarm, and the mean detection time can be found by putting $P_{1} \approx 0$ inEq.s(13), (14) and (24), respectively:

$$
\begin{aligned}
& \left.P_{d}=\left(P_{2} P_{d, E D}+\left(1-P_{2}\right)\left[P_{3} P_{4} P_{d, M F}+P_{3}\left(1-P_{4}\right) P_{d, C Y C}\right)+\left(1-P_{3}\right) P_{d, C Y C}\right]\right) \\
& \left.P_{f}=\left(P_{2} P_{f, E D}+\left(1-P_{2}\right)\left[P_{3} P_{4} P_{f, M F}+P_{3}\left(1-P_{4}\right) P_{f, C Y C}\right)+\left(1-P_{3}\right) P_{f, C Y C}\right]\right) \\
& \bar{T}=N\left(T_{c y c l \theta 1}\left[\left(1-P_{2}\right)\left(1-P_{3} P_{4}\right)\right]+T_{M F-1}\left[\left(1-P_{2}\right) P_{3} P_{4}\right]+T_{E D-1} P_{2}\right)+\bar{T}_{R}+\bar{T}_{S}
\end{aligned}
$$

Based on case 1 , especially when $P_{1} \approx 0$, the following two subcases can be made on the basis of $P_{2}$ :

Subcase 1.1: When $0.5 \leq P_{2} \leq 1$ for the majority of the channels, WB signal. Therefore, the CR will perform ED detection for sensing most of the channels. ED is suitable for detecting wideband signals. When $P_{2} \approx 1, \underline{(\text { Coop }-W B, P l=0, P 2=1)}$ then the probability of detection, the probability of false alarm, and the mean detection time can be found by putting $P_{2} \approx 1$ in Eq.s (25), (26) and (27), respectively:

$$
\begin{aligned}
& P_{d}=P_{d, E D} \\
& P_{f}=P_{f, E D} \\
& \bar{T}=N T_{E D-1}+\bar{T}_{R}+\bar{T}_{S}
\end{aligned}
$$

Subcase 1.2: When $0 \leq P_{2} \leq 0.5$ for the majority of the channels, NB signal. Therefore, the CR

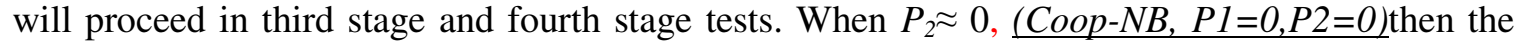
probability of detection, the probability of false alarm, and the mean detection time can be found by putting $P_{2} \approx 0$ in Eq.s (25), (26) and (27), respectively:

$$
\begin{aligned}
& P_{d}=P_{3} P_{4} P_{d, M F}+\left(1-P_{3} P_{4}\right) P_{d, C Y C} \\
& P_{f}=P_{3} P_{4} P_{f, M F}+\left(1-P_{3} P_{4}\right) P_{f, C Y C} \\
& \bar{T}=N\left(T_{\text {cyclel } 1}\left[\left(1-P_{3} P_{4}\right)\right]+T_{M F-1}\left[P_{3} P_{4}\right]\right)+\bar{T}_{R}+\bar{T}_{S}
\end{aligned}
$$

Based on Subcase 1.2, especially when $P_{2} \approx 0$, the following two subcases can be made on the basis of $P_{3}$ : 
Subcase 1.2.1: When $0 \leq P_{3} \leq 0.5$ for the majority of the channels, unknown signals. Therefore, the CR will not be able to detect most of signals by matched filter and hence, will perform

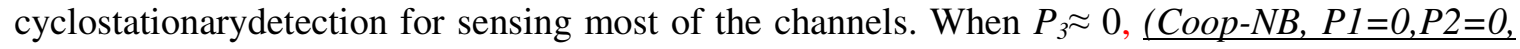

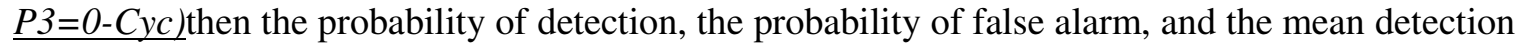
time can be found by putting $P_{3} \approx 0$ in Eq.s (31), (32) and (33), respectively:

$$
\begin{aligned}
& P_{d}=P_{d, C Y C} \\
& P_{f}=P_{f, C Y C} \\
& \bar{T}=N T_{c y c l \theta 1}+\bar{T}_{R}+\bar{T}_{S}
\end{aligned}
$$

Subcase 1.2.2: When $0.5 \leq P_{3} \leq 1$ for the majority of the channels; known signals. Therefore, the $\mathrm{CR}$ will be able to detect the majority of signals by MF if the SNIR is greater than T. Therefore, the CR will proceed in the fourth stage tests. When $P_{3} \approx 1,($ Coop $-N B, P 1=0, P 2=0, P 3=1)$ then the probability of detection, the probability of false alarm, and the mean detection time can be derived by putting $P_{3} \approx 1$ in Eq.s (31), (32) and (33), respectively:

$$
\begin{aligned}
& P_{d}=P_{4} P_{d, M F}+\left(1-P_{4}\right) P_{d, C Y C} \\
& P_{f}=P_{4} P_{f, M F}+\left(1-P_{4}\right) P_{f, C Y C} \\
& \bar{T}=N\left(T_{c y c l \text { l } 1}\left[\left(1-P_{4}\right)\right]+T_{M F-1}\left[P_{4}\right]\right)+\bar{T}_{R}+\bar{T}_{S}
\end{aligned}
$$

Based on Subcase 1.2.2, especially when $P_{3} \approx 1$, the following two subcases can be made on the basis of $P_{4}$ :

Subcase 1.2.2.1: When $0 \leq P_{4} \leq 0.5$ for the majority of the channels, the CR will perform cyclostationarydetection for sensing the majority of the channels since the SNIR is not suitable for using the matched filter. When $P \approx 0$, $(C O O P-N B, P 1=0, P 2=0, P 3=1, P 4=0, C Y C)$ and the probability of detection, the probability of false alarm, and the mean detection time can be derived by putting $P_{4} \approx 0$ in Eq.s (37), (38) and (39), respectively:

$$
\begin{aligned}
& P_{d}=P_{d, C Y C} \\
& P_{f}=P_{f, C Y C} \\
& \bar{T}=N T_{\text {cyclel }}+\bar{T}_{R}+\bar{T}_{S}
\end{aligned}
$$

Subcase 1.2.2.2: When $0.5 \leq P_{4} \leq 1$ for the majority of the channels, the SU will perform MFdetection for sensing the majority of the channels. The mean detection time of the MF detection is the least, and therefore the best case for the detection time is when the majority of channels are sensed by the MF. The best scenario is when $P_{4} \approx 0,(C O O P-N B, P 1=0, P 2=0$,

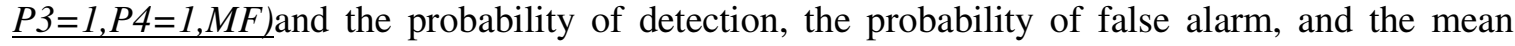
detection time can be found by putting $P_{4} \approx 1$ in Eq.s(37), (38) and (39), respectively: 
$P_{d}=P_{d, M F}$

$P_{f}=P_{f, M F}$

$\bar{T}=N T_{M F-1}+\bar{T}_{R}+\bar{T}_{S}$

Case 2: When $0.5 \leq P_{1}<1$ for the majority of the channels, $\mathrm{SNR} \geq \gamma_{\text {wall }}$ NON-COOP sensing). Therefore, the $\mathrm{CR}$ will perform noncooperative sensing and the algorithm will proceed in testing the bandwidth of the signal in the second stage. When $P_{1} \approx 1$, then the probability of detection, the probability of false alarm, and the mean detection time can be found by putting $P_{1} \approx 1$ in Eq.s ((13), (14) and (24), respectively:

$$
\begin{aligned}
& P_{d}=\left(P_{2} P_{d, E D}+\left(1-P_{2}\right) P_{3} P_{d, M F}+\left(1-P_{2}\right)\left(1-P_{3}\right) P_{d, E D}\right) \\
& P_{f}=\left(P_{2} P_{f, E D}+\left(1-P_{2}\right) P_{3} P_{f, M F}+\left(1-P_{2}\right)\left(1-P_{3}\right) P_{f, E D}\right) \\
& \bar{T}=N\left(T_{M F-1}\left[\left(1-P_{2}\right) P_{3}\right]+T_{E D-1}\left[P_{2}+\left(1-P_{2}\right)\left(1-P_{3}\right)\right]\right)
\end{aligned}
$$

Based on case 2 , especially when $P_{1} \approx 1$, the following two subcases can be made on the basis of $\mathrm{P} 2$, the probability of wide bandwidth of PU signal:

Subcase 2.1: When $0.5 \leq P_{2} \leq 1$ for the majority of the channels, the PU waveform is WB. Therefore, CR will perform noncooperativeED detection for sensing most of the channels. ED is suitable for detecting wideband signals. When $P_{2} \approx 1$, (noncoop-WB, $\left.P 1=1, P 2=1\right)$ then the probability of detection, the probability of false alarm, and the mean detection time can be found by putting $P_{2} \approx 1$ in Eq.s (46), (47) and (48), respectively:

$$
\begin{aligned}
& P_{d}=P_{d, E D} \\
& P_{f}=P_{f, E D} \\
& \bar{T}=N T_{E D 1}
\end{aligned}
$$

Subcase 2.2: When $0 \leq P_{2}<0.5$ for most of the channels, the PU waveform is NB. The CR will go to the third stage to test the signal knowledge. The probability of false alarm, and the mean detection time can be evaluated by putting $P_{2} \approx 0$ in Eq.s(46), (47) and (48), respectively:

$$
\begin{aligned}
& P_{d}=\left(P_{3} P_{d, M F}+\left(1-P_{3}\right) P_{d, E D}\right) \\
& P_{f}=\left(P_{3} P_{f, M F}+\left(1-P_{3}\right) P_{f, E D}\right) \\
& \bar{T}=N\left(T_{M F-1}\left[P_{3}\right]+T_{E D-1}\left[\left(1-P_{3}\right)\right]\right)
\end{aligned}
$$

Based on subcase 2.2, especially when $\left(P_{1} \approx 1, P_{2} \approx 0\right.$ ), the following two subcases can be made on the basis of $\mathrm{P}_{3}$, the probability of complete knowledge of PU signal. Note that the algorithm does not need to perform the fourth stage test since the SNIR is already greater than $\gamma_{\text {wall }}$. Which is greater than the threshold $\mathrm{T}$ : 
Subcase 2.2.1: When $0 \leq P_{3}<0.5$ for most of the channels, the PU waveform is unknown. Thenthe $\mathrm{CR}$ will perform noncooperativeenergy detection for sensing the majority of channels. When $P_{3} \approx$ 0 , all the signals are unknown, the probability of detection, the probability of false alarm, and the mean detection time can be evaluated by putting $P_{3} \approx 0$ in Eq.s(52), (53) and (54), respectively:

$$
\begin{aligned}
& P_{d}=P_{d, E D} \\
& P_{f}=P_{f, E D} \\
& \bar{T}=N T_{E D 1}
\end{aligned}
$$

Subcase 2.2.2: When $0.5 \leq P_{3}<1$ for most of the channels, the PU waveform is known. Then the CR will perform noncooperative matched filter for sensing the majority of channels. When $P_{3} \approx 1$, all the signals are known, the probability of detection, the probability of false alarm, and the mean detection time can be evaluated by putting $P_{3} \approx 1$ in Eq.s(52), (53) and (54), respectively:

$P_{d}=P_{d, M F}$

$P_{f}=P_{f, M F}$

$\bar{T}=N T_{M F-1}$

\section{Considered Setting ANd Simulation Parameters}

SimulationsusingMat-labwere carried out underthe following system setting: there are 8uniformly distributedPUs with signal, band and channelcharacteristics according to Table1.4 CRs seeking to sense the holes in the 8 PUs' bands whether individually (non-cooperatively)or using cooperative

\begin{tabular}{|c|c|}
\hline $\begin{array}{l}\text { BANDS OF } \\
\text { INTEREST }\end{array}$ & CHARACTERISTICS \\
\hline BAND-1 & $\begin{array}{c}\text { BW=5MHz, Channel: Multi-path, Carrier frequency: } 2 \mathrm{GHz} \\
\text { Signal type: WCDMA, SNIR }(\mathrm{dB})=-7\end{array}$ \\
\hline BAND-2 & $\begin{array}{c}\mathrm{BW}=7 \mathrm{MHz} \text {, Channel: Multi-path, Carrier frequency: } 5 \mathrm{GHz} \\
\text { Signal type: OFDM, SNIR }(\mathrm{dB})=0\end{array}$ \\
\hline BAND-3 & $\begin{array}{c}\text { BW=10MHz, Channel: Multi-path, Carrier frequency: } 5 \mathrm{GHz} \\
\text { Signal type: OFDM, SNIR(dB)=-17 }\end{array}$ \\
\hline BAND-4 & $\begin{array}{c}\text { BW=20MHz, Channel: Multi-path, Carrier frequency: } 8 \mathrm{GHz} \\
\text { Signal type: OFDM, SNIR }(\mathrm{dB})=-24\end{array}$ \\
\hline BAND-5 & $\begin{array}{c}\text { BW }=200 \mathrm{KHz} \text {, Channel: AWGN, Carrier frequency: } 900 \mathrm{MHz} \\
\text { Signal type: GMSK, SNIR(dB)=-3 }\end{array}$ \\
\hline BAND-6 & $\begin{array}{c}\text { BW }=100 \mathrm{KHz} \text {, Channel: AWGN, Number of samples: } 200 \mathrm{~K} \text {, Carrier } \\
\text { frequency: } 2 \mathrm{GHz} \\
\text { Signal type: } 16-\mathrm{QAM}, \mathrm{SNIR}(\mathrm{dB})=-19\end{array}$ \\
\hline BAND-7 & $\mathrm{BW}=50 \mathrm{KHz}$, Channel: AWGN, Carrier frequency: $2 \mathrm{GHz}$ \\
\hline
\end{tabular}
detection where CR-1 acts as the fusion center. The simulation parameters are given in Table 2.

Table 1: Characteristics of bands of interest 
International Journal of Wireless \& Mobile Networks (IJWMN) Vol. 8, No. 4, August 2016

\begin{tabular}{|c|c|}
\hline & Signal type: 4-QAM, SNIR $(\mathrm{dB})=10$ \\
\hline BAND-8 & $\mathrm{BW}=30 \mathrm{KHz}$, Channel: AWGN, Carrier frequency: $400 \mathrm{KHz}$ \\
Signal type: BPSK, SNIR $(\mathrm{dB})=-21$
\end{tabular}

Table 2: Simulation parameters for considered setting

\begin{tabular}{|c|c|}
\hline Parameter & Value/Assumption \\
\hline Number of Bands & 8 \\
\hline $\begin{array}{l}\text { Probability of false alarm for each } \\
\text { detection scheme }\end{array}$ & 0.01 \\
\hline $\begin{array}{l}\text { Sensing times for a single channel } \\
\text { by } \\
\text { CS }\left(T_{1}\right) \text {, con-ED }\left(T_{2}\right) \text { and } \operatorname{MF}\left(T_{3}\right)\end{array}$ & $\begin{array}{c}T_{1}=12 \mathrm{msec}, T_{2}=2 \mathrm{msec} \\
\text { and } T_{3}=1 \mathrm{msec} \text { Error! } \\
\text { Reference source not } \\
\text { found. }\end{array}$ \\
\hline SNR wall & $\begin{array}{l}-9 \mathrm{~dB} \\
{[21]}\end{array}$ \\
\hline $\mathrm{T}_{\mathrm{R}}$ & $3 \operatorname{ms}(\max )$. \\
\hline $\mathrm{T}_{\mathrm{S}}$ & $1 \mathrm{~ms}$ (max.) \\
\hline FFT POINTS & 256 \\
\hline $\begin{array}{l}N_{f} N_{t} \\
\text { Nf and Nt are the averaging filter } \\
\text { lengths in the frequency and time } \\
\text { domain, respectively }\end{array}$ & $\begin{array}{l}N_{f}=4 \\
N_{t=40}\end{array}$ \\
\hline$T_{m f}$ & $-18 \mathrm{~dB}[21]$ \\
\hline
\end{tabular}

\section{NUMERICAL RESULT}

Table 3 shows the main characteristics of each band of interest based on which the proposed adaptive algorithm selects the appropriate detection scheme individually or cooperatively. The figure also indicates the applicability or inapplicability of other schemes besides the selected one. It is shown that for the four wideband signals, SBED is always selected as all other schemes are inapplicable to sense wideband. For narrow band signals, MF is selected when the signal characteristics are known enough and OOCS is selected according otherwise. 
International Journal of Wireless \& Mobile Networks (IJWMN) Vol. 8, No. 4, August 2016

Table 3: Adaptive Algorithm: Selected Scheme and applicability of other Schemes

\begin{tabular}{|c|c|c|c|c|c|c|c|c|c|}
\hline \multicolumn{6}{|c|}{ PU Signal and Bandwidth Characteristics } & \multicolumn{4}{|c|}{$\begin{array}{l}\text { Adaptive Algorithm: Selected Scheme } \\
\text { and applicability of other Schemes }\end{array}$} \\
\hline $\begin{array}{l}\text { BANDS } \\
\text { OF } \\
\text { INTERES } \\
T \\
\end{array}$ & BW & $\begin{array}{c}\text { Chan } \\
\text { nel }\end{array}$ & $\begin{array}{l}\text { Signal } \\
\text { Type }\end{array}$ & $\begin{array}{c}\text { SNIR } \\
(\mathrm{dB})\end{array}$ & $\begin{array}{c}\text { Signal } \\
\text { knowled } \\
\text { ge }\end{array}$ & $\begin{array}{c}\text { Select } \\
\text { ed } \\
\text { schem } \\
\text { e }\end{array}$ & SBED & MF & OOCS \\
\hline BAND-1 & $\begin{array}{l}5 \mathrm{M} \\
\mathrm{Hz}\end{array}$ & $\begin{array}{c}\text { Multi } \\
\text {-path }\end{array}$ & $\begin{array}{c}\text { WCDM } \\
\mathrm{A}\end{array}$ & -7 & known & $\begin{array}{l}\text { Non- } \\
\text { coop. } \\
\text { SBED }\end{array}$ & & $\begin{array}{l}\text { Inapplicable } \\
\mathrm{X}\end{array}$ & $\begin{array}{l}\text { Inspplicable } \\
\mathrm{X}\end{array}$ \\
\hline BAND-2 & $\begin{array}{l}7 \mathrm{M} \\
\mathrm{Hz}\end{array}$ & $\begin{array}{c}\text { Multi } \\
\text {-path }\end{array}$ & OFDM & 0 & Unknown & $\begin{array}{l}\text { Non- } \\
\text { coop. } \\
\text { SBED }\end{array}$ & & $\begin{array}{l}\text { Inapplicable } \\
\mathrm{X} \\
\end{array}$ & $\begin{array}{l}\text { Inspplicable } \\
\mathrm{X}\end{array}$ \\
\hline BAND-3 & $\begin{array}{l}10 \mathrm{M} \\
\mathrm{Hz}\end{array}$ & $\begin{array}{c}\text { Multi } \\
\text {-path }\end{array}$ & OFDM & -17 & known & $\begin{array}{l}\text { Coop. } \\
\text { SBED }\end{array}$ & & $\begin{array}{c}\text { Inapplicable } \\
\mathrm{X} \\
\end{array}$ & $\begin{array}{c}\text { Inapplicable } \\
\mathrm{X}\end{array}$ \\
\hline BAND-4 & $\begin{array}{c}20 \mathrm{M} \\
\mathrm{Hz}\end{array}$ & $\begin{array}{c}\text { Multi } \\
\text {-path }\end{array}$ & OFDM & -24 & Unknown & $\begin{array}{l}\text { Coop. } \\
\text { SBED }\end{array}$ & & $\begin{array}{l}\text { Inapplicable } \\
\mathrm{X}\end{array}$ & $\begin{array}{l}\text { Inspplicable } \\
\mathrm{X}\end{array}$ \\
\hline BAND-5 & $\begin{array}{l}200 \\
\mathrm{KHz}\end{array}$ & $\begin{array}{c}\text { AWG } \\
\mathrm{N}\end{array}$ & GMSK & -3 & known & $\begin{array}{l}\text { Non- } \\
\text { coop. } \\
\text { MF }\end{array}$ & Applicable & & Applicable \\
\hline BAND-6 & $\begin{array}{c}100 \\
\mathrm{KHz}\end{array}$ & $\begin{array}{c}\text { AWG } \\
\mathrm{N}\end{array}$ & $\begin{array}{c}16- \\
\text { QAM }\end{array}$ & -19 & Unknown & $\begin{array}{l}\text { Coop. } \\
\text { OOCS }\end{array}$ & $\begin{array}{c}\text { Inapplicable } \\
\mathrm{X} \\
\end{array}$ & $\begin{array}{l}\text { Inapplicable } \\
\mathrm{X} \\
\end{array}$ & \\
\hline BAND-7 & $\begin{array}{l}50 \mathrm{~K} \\
\mathrm{~Hz}\end{array}$ & $\begin{array}{c}\text { AWG } \\
\mathrm{N}\end{array}$ & 4-QAM & 10 & known & $\begin{array}{l}\text { Non- } \\
\text { coop. } \\
\text { MF }\end{array}$ & Applicable & & Applicable \\
\hline BAND-8 & $\begin{array}{l}30 \mathrm{~K} \\
\mathrm{~Hz}\end{array}$ & $\begin{array}{c}\text { AWG } \\
\mathrm{N}\end{array}$ & BPSK & -21 & Unknown & $\begin{array}{l}\text { Coop. } \\
\text { OOCS }\end{array}$ & $\begin{array}{l}\text { Inapplicable } \\
\mathrm{X}\end{array}$ & $\begin{array}{c}\text { Inapplicable } \\
\mathrm{X}\end{array}$ & \\
\hline
\end{tabular}

Based on Table 3, Figure 3 shows the probabilities of detection for each band in interest according to the selected detection scheme and other applicable ones. Conventional energy detection, although not used in the algorithm, is added to the figure in order to compare it with SBED. The figure shows that optimal probability of detection (1.00) at band-1 using SBED when SNIR is $0 \mathrm{~dB}$ but it is better at band-3 than at band-1 although the signal there has lower SNIR. That was due to the use of cooperative sensing. In band-4, the probability of detection is below 0.9 due to very low SNIR despite using cooperative sensing. The narrowband signals in band 5to- 8 , MF is used when the signal is known and OOCS is used otherwise. We note that the conventional energy detection, if used, will perform well in band-5 and band-7 where the SNIR is relatively adequate for its function. However, for band- 6 and band-8, its probabilities of detection are very poor due to inadequate SNIR. 


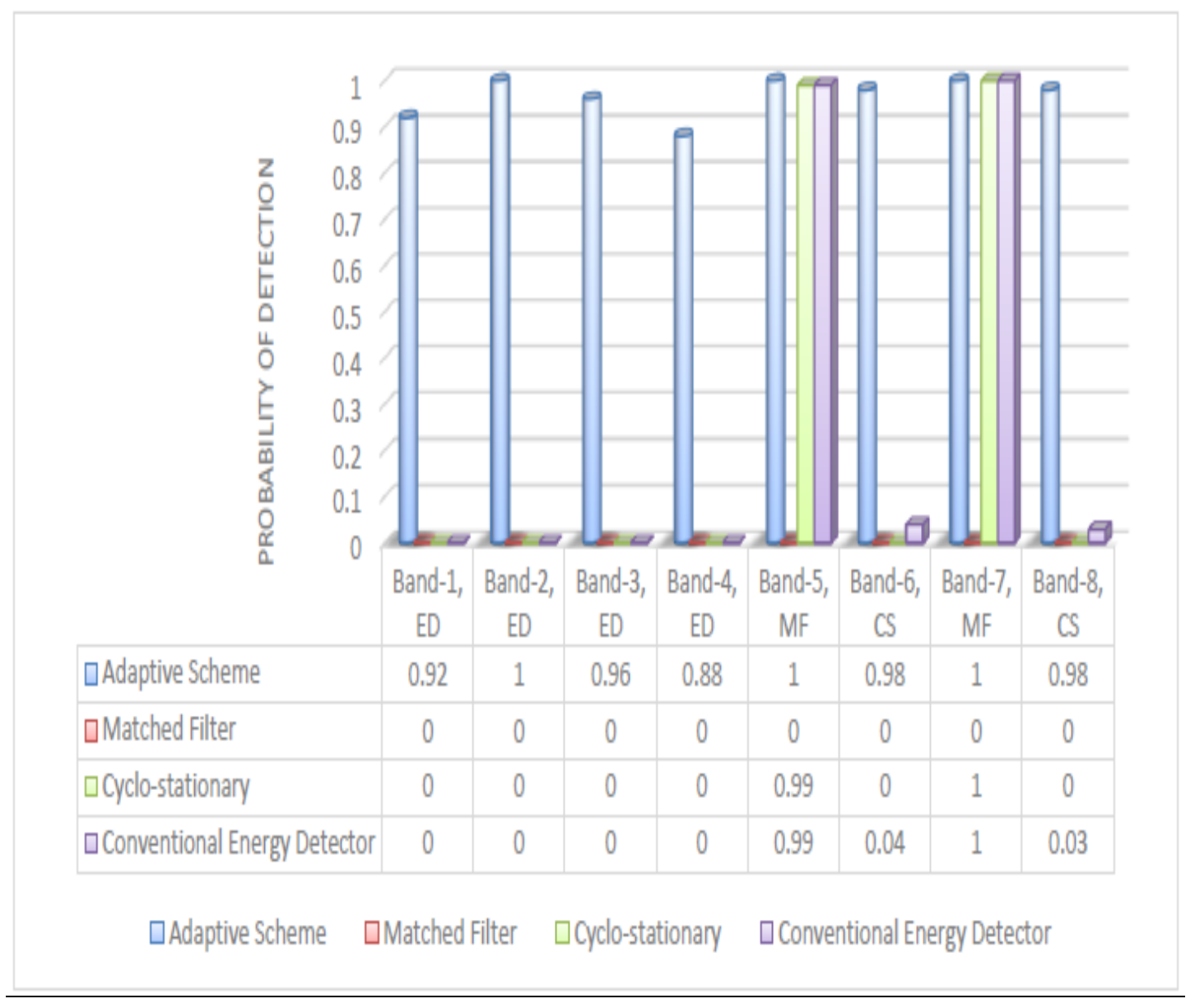

Figure 3: Probabilities of detection for each band in interest according to the selected detection scheme and other applicable ones.

Figure 4 shows the mean detection times for each band in interest according to the selected detection scheme and other applicable ones. We notice relatively long detection times for wideband signals as expected. However, for narrow band ones, it depends on the selected detection scheme and whether cooperative or individual detection. Note that cooperative overheads are represented by extra reporting and synchronization delays in addition to the sensing time. Comparing with results achieved in Figure 3, we notice that the selected scheme for both band-5 and band-7 (MF) was the best as it yields best probability of detection and least detection time. However, for both band-6 and band-8, cooperative OOCS was selected since complete signal characteristics are unknown and suffers from low SNIR. So, no way to use MF for lower detection time. Moreover, if conventional ED was used, it would lead to very low probability of detection. 
International Journal of Wireless \& Mobile Networks (IJWMN) Vol. 8, No. 4, August 2016

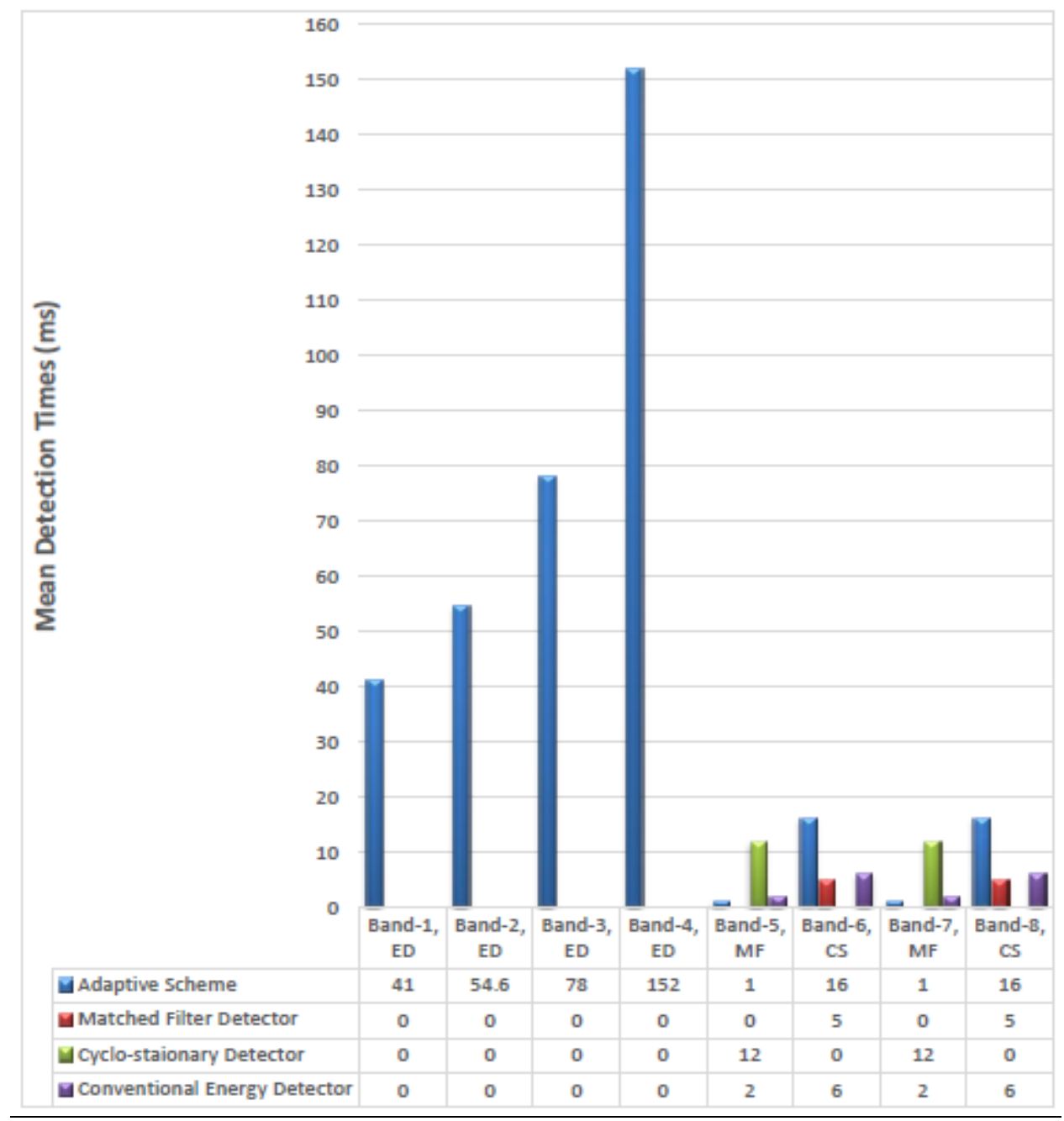

Figure 4: Mean detection times for each band in interest according to the selected detection scheme and other applicable ones.

Figure 5 shows the average probability of detection- averaged over all bands- for different values of $P_{1}$ (the probability that channel would be sensed by the non-cooperative detections) under the condition that each of other probabilities, $P_{2}, P_{3}$ and $P_{4}$, is fixed to 0.5. Figure 5 shows the average detection time under same conditions. 
International Journal of Wireless \& Mobile Networks (IJWMN) Vol. 8, No. 4, August 2016

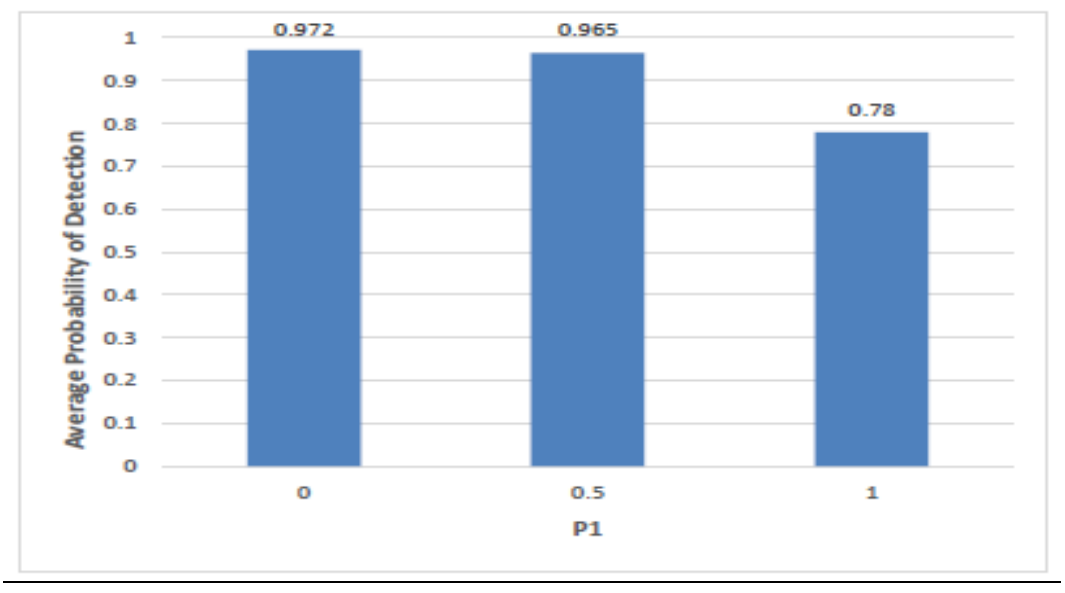

Figure 5: Average probability of detection for different values of $P_{1}$, average SNIR=10dB

The two figures show that when $P_{1}=0$, which mean all the band are sensed cooperatively, the average probability of detection is 0.972 at the expense of $48.2 \mathrm{~ms}$ of average detection time. At the other edge, if $P_{1}=1$, which mean all the bands are sensed non-cooperatively, the average probability of detection becomes practically unacceptable (0.78) although the average detection time is lower due to the absence of reporting and synchronization delays. The best case was when $P_{1}=0.5$ where half of the bands are sensed cooperatively and the other half are sensed individually as the case of the proposed adaptive model.

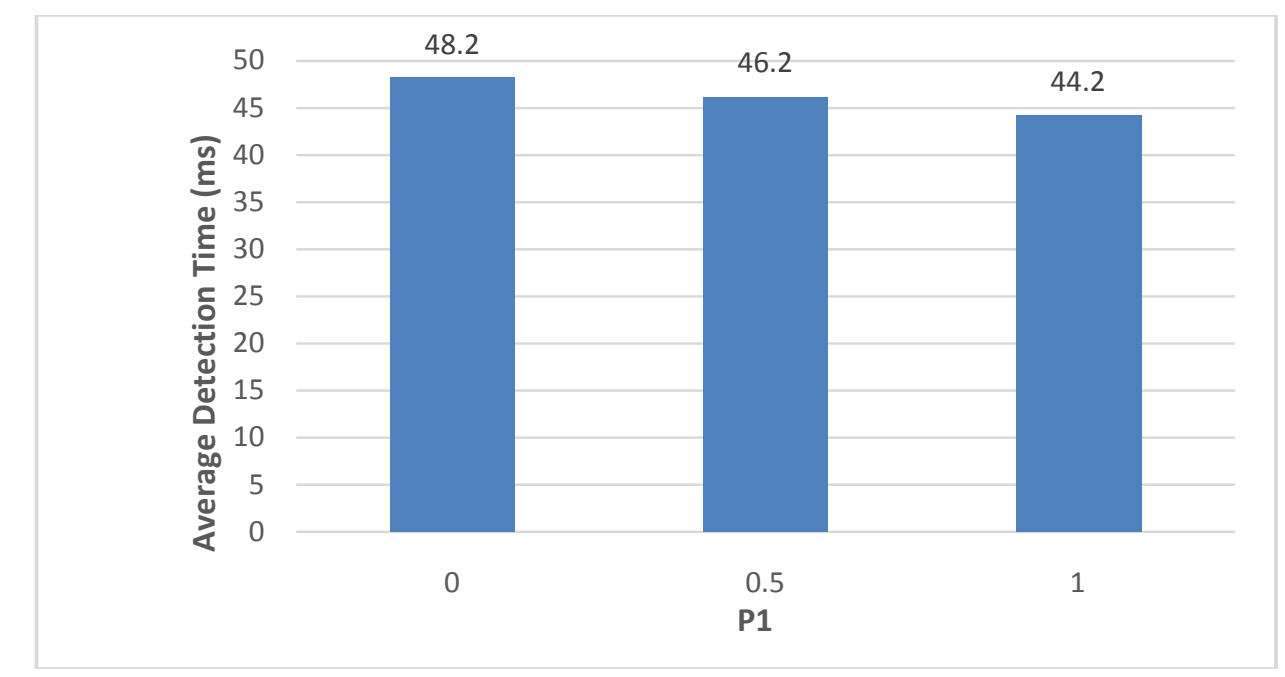

Figure 6: Average detection times for different values of $P_{1}$, average SNIR=10dB 
In Table 3, the performance of the proposed multi-stage detection scheme is compared with existing onespresented in Section 1. As mentioned in section 1, no other scheme considered switching between local and cooperative sensing for most types of PUs' signals with both narrow and wide bands- reaching $20 \mathrm{MHz}$.Moreover, signals experience practical wireless channels including hidden PU problem. Although comparison is not fair, the results of proposed schemeindicatesthat the probability of detection is around 0.965 for a given false alarmprobability of 0.1 at an assumed average SNR of $-10 \mathrm{~dB}$.Although some other schemes, show slightly better detection probabilities, however, if they encountered the aforementioned conditions of the proposed one, their performance would be different.

Table 4: Performance Comparison with Existing Sensing Schemes

\begin{tabular}{|c|c|c|c|c|}
\hline $\begin{array}{l}\text { Detection } \\
\text { Scheme }\end{array}$ & $\begin{array}{l}\text { Avera } \\
\text { ge } \\
\text { SNR } \\
\text { (dB) }\end{array}$ & $\boldsymbol{P}_{f}$ & $\boldsymbol{P}_{d}$ & Applicability \\
\hline $\begin{array}{l}\text { Proposed } \\
\text { Scheme }\end{array}$ & -10 & 0.1 & 0.965 & 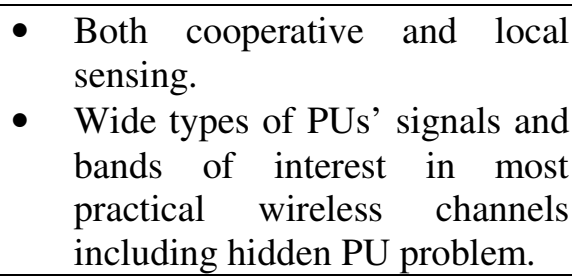 \\
\hline I3S[6] & -10 & 0.1 & 0.99 & $\begin{array}{ll}\text { - } & \text { Local sensing } \\
\text { - } & \text { No hidden PU problem. } \\
\text { - } & \text { Only Gaussian channels. }\end{array}$ \\
\hline $\begin{array}{l}\text { Fuzzy Logic- } \\
\text { Based[10] }\end{array}$ & -10 & $\begin{array}{l}0.00 \\
01\end{array}$ & 0.97 & 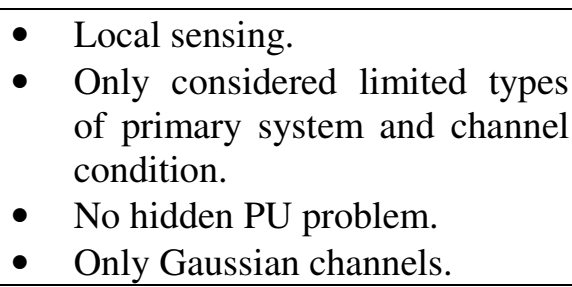 \\
\hline $\begin{array}{l}\text { SNR-based } \\
\text { adaptive[5] }\end{array}$ & -10 & 0.1 & 0.75 & $\begin{array}{l}\text { - Local sensing. } \\
\text { - Only considered limited types } \\
\text { of primary system and channel } \\
\text { condition. } \\
\text { - No hidden PU problem. }\end{array}$ \\
\hline $\begin{array}{l}\text { High-speed } \\
\text { two-stage[11] }\end{array}$ & -16 & 0.1 & 0.9 & 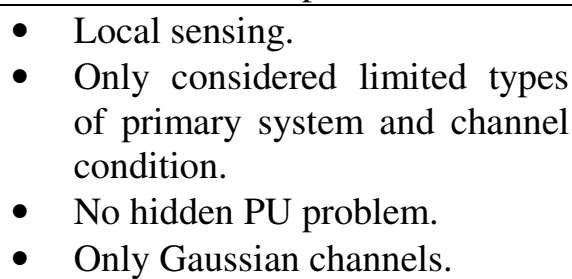 \\
\hline
\end{tabular}




\begin{tabular}{|l|l|l|l|ll|}
\hline $\begin{array}{l}\text { Fast two- } \\
\text { stage[12] }\end{array}$ & -11 & 0.1 & 0.93 & $\begin{array}{l}\bullet \\
\bullet\end{array}$ & $\begin{array}{l}\text { Local sensing. } \\
\text { Only considered limited types } \\
\text { of primary system and channel } \\
\text { condition. } \\
\text { No hidden PU problem. }\end{array}$ \\
\hline
\end{tabular}

\section{CONCLUSION}

The proposed multi-stage detection scheme deals withalmost all types of PUs' signals and bands of interest. It tries to detect signals experiencing practical wireless channels and decides the detection algorithm based on the power, noise, bandwidth and knowledge of signal, thus trading off accuracy and mean detection time for the overall detection process. The performance of the proposed multi-stage scheme is compared with existing onesin spite of the fact that no one of them considered most types of PUs' signalswith both narrow and wide bands- reaching $20 \mathrm{MHz}$, as the proposed scheme did. Moreover,detecting signals experiencing practical wireless channels including hidden PU problem necessitate switching between local and cooperative sensing. The results of proposed scheme indicate that the probability of detection is around 0.965 for a given of false alarmprobability of 0.1 at an assumed average SNR of $-10 \mathrm{~dB}$. Although some other schemes, show slightly better detection probabilities, however, if they encountered the aforementioned conditions of the proposed one, their performance would be different.

\section{References}

[1] J. Kantiand G. Singh Tomar "Various Sensing Techniques in Cognitive Radio Networks: A Review" International Journal of Grid and Distributed Computing Vol. 9, No. 1 (2016), pp.145-154.

[2] SenerDikmese, Paschalis C. Sofotasios, Terolhalainen, Markku Renfors and MikkoValkama, "Efficient Energy Detection Methods for Spectrum Sensing under Non-Flat Spectral Characteristics," IEEE Journal on Selected Areas in Communications (JSAC), DOI:10.1109/JSAC.2014.236-1074, Oct. 2014.

[3] W. Yue, B. Zheng, and Q. Meng, "Cyclostationary property based spectrum sensing algorithms for primary detection in cognitive radio systems," Journal of Shanghai Jiaotong University (Science), vol. 14, no. 6, pp. 676-680, Dec. 2009

[4] H. Sun et al., "Wideband spectrum sensing for cognitive radio networks- a survey" IEEE Wireless Communications • April 2013, pp 74-81

[5] Ejaz et al. " SNR-based adaptive spectrum sensing for cognitive radio networks". International Journal of Innovative Computing, Information and Control Vol. 8, No: 9, 6095-6106 (2012)

[6] Ejaz et al. " I3S: Intelligent spectrum sensing scheme for cognitive radio networks" EURASIP Journal onWireless Communications and Networking 2013, 2013:26

[7] Amardip Kumar et al. "An Adaptive and Efficient Local Spectrum Sensing Scheme in Cognitive Radio Networks" International Journal of Computer Applications (0975 - 8887) Volume72- No.23, June 2013

[8] W Yue, B Zheng, Q Meng, W Yue, Combined energy detection one-order cyclostationary feature detection techniques in cognitive radio systems. J China Univ. Posts Telecommun. 17(4), 18-25 (2010)

[9] K. Srisomboo et al., "Two-stage Spectrum Sensing for Cognitive Radio under Noise Uncertainty" Proceeding of the Eighth International Conference on Mobile Computing, 2015, pp 19-24.

[10] WEjaz, NU Hasan, MA Azam, HS Kim, Improved local spectrum sensing for cognitive radio networks. EURASIP J. Adv. Signal Process(2012).http:// asp.eurasipjournals.com/content/2012/1/242 
International Journal of Wireless \& Mobile Networks (IJWMN) Vol. 8, No. 4, August 2016

[11] S Geethu, GL Narayanan, A novel high speed two stage detector for spectrum sensing. Elsevier Procedia Technol.6,682-689(2012)

[12] PR Nair, APVinod, KGSmitha, AKKrishna, Fast two-stage spectrum detector for cognitive radios in uncertain noise channels. IET Commun. 6(11),1341-1348(2012)

[13] Akyildiz, Ian F. et al.,"Cooperative spectrum sensing in cognitive radio networks: A survey", Physical Communication 4 (2011) 40-62.

[14] Lamiaa Khalid and AlaganAnpalagan, "Adaptive Assignment of Heterogeneous Users for GroupBased Cooperative Spectrum Sensing” IEEE TRANSACTIONS ON WIRELESS COMMUNICATIONS, VOL. 15, NO. 1, pp 232-246, 2016.

[15] J. So, T. Kwon "Limited reporting-based cooperative spectrum sensing for multiband cognitive radio networks" Int. J. Electron. Commun. (AEÜ) 70 (2016) 386-397

[16] W.-Y. Lee, I.F. Akyildiz, Optimal spectrum sensing framework for cognitive radio networks, IEEE Transactions on Wireless Communications 7 (10) (2008) 3845-3857.

[17] E. Peh, Y.-C. Liang, Y.L. Guan, Y. Zeng, Optimization of cooperative sensing in cognitive radio networks: a sensing-throughput tradeoff view, IEEE Transactions on Vehicular Technology 58 (9) (2009) 5294-5299.

[18] A. Ghasemi, E.S. Sousa, Spectrum sensing in cognitive radio networks: the cooperation-processing tradeoff, Wireless Communicationsand Mobile Computing 7 (9) (2007) 1049-1060.

[19] X. Zhou, J. Ma, G. Li, Y. Kwon, A. Soong, Probability-based combination for cooperative spectrum sensing, IEEE Transactions on Communications 58 (2) (2010) 463-466.

[20] Tandra, R., et al.,"SNR walls for signal detection", IEEE J. Sel. Topics Signal Process., 2008, 2, (1), pp. 4-17.

[21] Amardip Kumar et al. "An Adaptive and Efficient Local Spectrum Sensing Scheme in Cognitive Radio Networks" International Journal of Computer Applications (0975 - 8887) Volume72- No.23, June 2013

[22] W. Yue, B. Zheng, Q. Meng and W. Yue, Combined energy detection and one-order cyclostationary feature detection techniques in cognitive radio systems, The Journal of China Universities of Posts and Telecommunications, vol.17, no.4, pp.18-25, 2010.

[23] IEEE Computer Society, IEEE Std 802.22-2011 Part 22: Cognitive Wireless RAN Medium Access Control (MAC) and Physical Layer (PHY) Specifications: Policies and Procedures for Operation in the TV Bands. IEEE Standard for Information Technology, 1-672 (2011) 